\title{
Effect of Double Ruler Magnetic Field in Controlling Meniscus Flow and Turbulence Intensity Distribution in Continuous Slab Casting Mold
}

\author{
Sandip SARKAR, ${ }^{*}$ Vikas SINGH, Satish Kumar AJMANI, Ravi RANJAN and Kalyansundaram RAJASEKAR
}

Tata Steel Limited, Jamshedpur-831001, India.

(Received on May 31, 2016; accepted on August 4, 2016; J-STAGE Advance published date: November 12, 2016)

\begin{abstract}
To find the effect of double ruler electromagnetic breaking $(\mathrm{EMBr})$ in continuous casting flow control (FC) mold, present investigation aims at developing a validated transient magnetohydrodynamic (MHD) flow and turbulence model for the FC mold caster of the Tata Steel plant. Towards the above, the mathematical model firstly validated with the online plant measurement data and found to be in good agreement. Based on the plant measurement of magnetic fields at various EMBr ruler current settings, simulations are carried out for different casting speeds and section sizes. Heavy meniscus flow instabilities during $\mathrm{EMBr}$ OFF conditions are found to be suppressed through the retarding influence of applied magnetic field. The effect of EMBr causes thinning of the velocity boundary layer and the flow is found more prone to be surface directed. In the mold, magnetic field dampens turbulence and emboldens the formation of large scale vertical structures, whose axis is aligning with the magnetic field vector. The jet hitting the narrow wall becomes more focused and reduced lateral dispersion during EMBr ON condition. Change in $\mathrm{EMBr}$ ruler positions leading to a shallower downward jet angle and higher surface velocity.
\end{abstract}

KEY WORDS: EMBr; mold; casting; steelmaking; flow field.

\section{Introduction}

During the continuous casting of the steel, the quality of cast products is predominantly affected by the flow dynamics at the meniscus level of the mold. An efficient caster operation ensures not only stable flow conditions and associated steel quality, but also meets the requirements for the steady operation without serious fluctuations in the casting process. The global flow characteristic is the function of mold thickness, mold width, argon injection rate, casting speed, and geometrical configuration of submergence entry nozzle (SEN). ${ }^{1)}$ Furthermore, abrupt enhancement in mold surface velocity variation brings asymmetry on the surface directed flow and its later effect passes to the formation of localized vortex and therefore occurrences of instability between the molten steel and slag layer interface. As a result, intermittent slag layer further entrained into the molten pool to form internal and surface defects in the final cast products. In contrast, irregular sluggish surface flow could turn into a low and non-uniform surface temperature. ${ }^{2)}$ This induces inadequate meniscus freezing, slag melting and infiltration, hook formation, and surface defects as consequent to the initial solidification issues. In the recent past, it has been observed by the various steel industries across the globe that application of electromagnetic fields may potentially be able to be accustomed to respond to the fluctuations in the flow. Furthermore, the local turbulent flow can be dampened instantaneously with the electromagnetic forces. ${ }^{3)}$

In continuous casting of the steel, Electromagnetic brake

* Corresponding author: E-mail: sandip.sarkar@tatasteel.com

DOI: http://dx.doi.org/10.2355/isijinternational.ISIJINT-2016-313
$(\mathrm{EMBr})$ is a practice of imposing a static magnetic field on the mold region of a steel continuous caster. Traditionally, electromagnetic brakes employ separate coils with direct current to generate a static magnetic field, which affect the flow in the mold cavity. In an effort to create rectangular regions of magnetic field near the SEN ports, design considerations of EMBr employs two magnets on each wide side of the mold. The induced magnetic field becomes aligned perpendicular to the main flow direction, and as a result of the interaction between the electrically conductive molten steel inside the mold cavity, a force is generated in the opposite direction of fluid flow. ${ }^{4}$ This result in a retarding force to slow down the velocity of the steel jet released from the ports of the submerged entry nozzle and thereby leading to damp strong velocity fluctuations inside the mold. In practice, the applied EMBr fields consist wide "ruler-shaped" magnetic field, double-ruler fields, and cylindrical-shaped fields through the entire width of the mold. Apart from static field (DC), alternating current (AC) fields are sometimes used for electromagnetic stirring (EMS) applications. There are few other applications where "multimode EMS" is used as a level accelerator (EMLA) and an electromagnetic level stabilizer (EMLS) for decelerating flow.,6)

There have been various literatures emerged in the recent past where researchers used different orientations of magnetic field to study the flow behaviour in the mold. In most of the cases, researchers used numerical techniques to model the governing flow and magnetic field interactions; however, there are few instances where experimental techniques have been used. ${ }^{4)}$ Computational techniques devoted in this endeavour mainly used several turbulence models and its effects on the final results of magnetohydrodynamic 
flows. ${ }^{7)}$ In those studies, both steady and unsteady governing equations are solved for the mold geometry. Among those, in most of the cases, researchers used steady magnetohydrodynamic equations to capture overall flow dynamics. In comparison, studies on the transient flow effects of EMBr are relatively scarce. ${ }^{7-9)}$

Qian and $\mathrm{Wu}^{10)}$ simulated Large Eddy Simulations (LES) computations to study the influence of submerged entry nozzle (SEN) depth, EMBr, and port angle on the vorticity and flow dynamics in the continuous slab casting. In another study, Kageyama and Evans ${ }^{11)}$ carried out a coupled LES-electromagnetic field with a mold free surface model to investigate the effect of electromagnetic forces on global flow kinetics. Effects of double ruler magnetic field on turbulent fluctuations and flow stability have been reported by Miki and Takeuchi. ${ }^{12)}$ Recently, Chaudhary et $a l .{ }^{13)}$ reported the effect of electromagnetic ruler braking (EMBr) on transient turbulent flow in continuous slab casting using LES. Singh et al. ${ }^{14)}$ carried out transient LES computations in a continuous slab casting mounted with double-ruler electromagnetic field.

In the present investigation, a numerical study has been performed in a real commercial caster of Tata Steel LD2 plant to investigate the effect of double-ruler EMBr magnetic field on the turbulent transient flow dynamics at various casting speeds, section sizes, and current settings. Results have been shown for both EMBr OFF and EMBr $\mathrm{ON}$ conditions. Actual $\mathrm{EMBr}$ magnetic field distribution data for numerical simulations have been generated through direct plant measurement. Model validation is performed through nail-board experimental data at plant. Furthermore, effect of changing ruler positions is also analyzed for possible modifications of the existing mold.

\section{Mathematical Modelling}

\subsection{Computational Geometry of the Model Caster}

As described above, the present investigation aims at the development of in-house full phased MHD model for slab caster of LD plant at Tata Steel. Towards this, three specified plant operating cases have been adopted as per the section sizes and submergence depths. The relevant section sizes considered in the current investigations are $1000 \mathrm{~mm} \times$ $218 \mathrm{~mm}, 1300 \mathrm{~mm} \times 218 \mathrm{~mm}$, and $1500 \mathrm{~mm} \times 218 \mathrm{~mm}$, respectively. In each case, the submergence depths have been fixed at $160 \mathrm{~mm}$. Since unsteady computations have been carried out in the present investigation, a mold length of $5000 \mathrm{~mm}$ has been chosen to accommodate two recirculation vortices being shedded from the primary recirculation zone.

\subsection{Governing Equations for Magnetohydrodynamic} Flows and Boundary Conditions

In the present investigation, the assumptions invoked in the present analysis are: the flow is Newtonian, incompressible, and unsteady; magnetic Reynolds number (defined as the ratio between magnetic advection to the magnetic diffusion, $R e_{m}$ ) is less than one, which is true for liquid steel; electric potential is coupled with Maxwell stress; the magnetohydrodynamic induced Lorenz force is acting perpendicular to the flow direction; and the Hartmann layer is negligible compared to turbulent boundary layer. It is wellknown that when an electrically conducting material moves through a magnetic field, an electric current is induced. This induced electric current interacts with the magnetic field and generates a force $\left(J \times B_{0}\right)$ on the flow field, called the Lorentz force. This Lorentz force breaks the flow and therefore opposes the very mechanism that created it. The following equations mathematically describe the governing flow evolution for an incompressible MHD flow: ${ }^{15)}$

$$
\text { Continuity : } \nabla \cdot \vec{u}=0
$$

$$
\text { Momentum : } \rho\left(\frac{\partial \vec{u}}{\partial t}+\vec{u} \cdot \nabla \vec{u}\right)=-\nabla p+\mu \nabla^{2} \vec{u}+\overrightarrow{F_{L}} \ldots
$$

Here, $\rho$ is the fluid density, $\vec{u}$ is the velocity vector, $p$ is the pressure, $\mu$ is the fluid viscosity, and $\overrightarrow{F_{L}}$ is the Lorentz force vector. ${ }^{15)}$

Turbulence: Standard $k-\varepsilon$

Turbulence kinetic energy :

$$
\frac{\partial(\rho k)}{\partial t}+\nabla \cdot(\rho u k)=\nabla \cdot\left[\Gamma_{k} \nabla k\right]+G-\rho \varepsilon
$$

Turbulence dissipation rate :

$$
\begin{array}{r}
\frac{\partial(\rho \varepsilon)}{\partial t}+\nabla \cdot(\rho u \varepsilon)=\nabla \cdot\left[\Gamma_{\varepsilon} \nabla \varepsilon\right]+\frac{\varepsilon}{k}\left(C_{1} G-C_{2} \rho \varepsilon\right) \\
\text { Turbulence viscosity : } \mu_{t}=C_{\mu} \rho \frac{k^{2}}{\varepsilon} \ldots \ldots \ldots . . .
\end{array}
$$

Where $\Gamma_{k}$ and $\Gamma_{\varepsilon}$ are the diffusion coefficients for the turbulent kinetic energy and its dissipation rate, respectively, and are given by ${ }^{16}$

$$
\Gamma_{k}=\frac{\mu_{e f f}}{\sigma_{k}} \Gamma_{\varepsilon}=\frac{\mu_{e f f}}{\sigma_{\varepsilon}} .
$$

Where $\mu_{\text {eff }}$ is the effective viscosity and is given by $\mu_{\text {eff }}=$ $\mu_{l}+\mu_{t}$

The tensor expression for the generation term $G$ is given as

$$
G=\mu_{t} \frac{\partial u_{j}}{\partial x_{i}}\left(\frac{\partial u_{j}}{\partial x_{i}}+\frac{\partial u_{i}}{\partial x_{j}}\right)
$$

Values for $C_{\mu}, C_{1}, C_{2}, \sigma_{k}, \sigma_{h}$, and $\sigma_{\varepsilon}$ are $0.09,1.44,1.92$, $1.0,0.9$, and 1.3 , respectively. ${ }^{16)}$

Since magnetic Reynolds number $\left(R e_{m}\right)$ is less than unity for liquid metals, the induced magnetic field due to the induced electric current can be neglected. After neglecting the induced magnetic field, the electric potential method can be used to determine the induced current and the Lorentz force by the following set of Maxwell's equations. ${ }^{13-15)}$

$$
\text { Lorentz force : } \overrightarrow{F_{L}}=\vec{J} \times \overrightarrow{B_{0}}
$$

Ohm's law: $\vec{J}=\sigma\left(\vec{E}+\vec{u} \times \overrightarrow{B_{0}}\right)=\sigma\left(-\nabla \phi+\vec{u} \times \overrightarrow{B_{0}}\right)$

$$
\text { Charge conservation : } \nabla \cdot \vec{J}=0
$$

Here, $\vec{J}$ is the electric current density vector, $\sigma$ is electrical conductivity, $\phi$ is electric potential, and $\vec{E}$ is the induced electric field.

By inserting current from Eq. (5b) into the conservation of charge Eq. (5c), a Poisson equation for electric potential can be derived as, ${ }^{13)}$

$$
\nabla^{2} \phi=\nabla \cdot\left(\vec{u} \times \overrightarrow{B_{0}}\right)
$$

where the external magnetic field vector is given as: $\vec{B}=\left(B_{0 x}, B_{0 y}, B_{0 z}\right)$.

As per as the boundary conditions are concerned, at the mold inlet the mean velocity is assumed to be uniform though its cross section and is specified with a velocity value conforming to a typical casting speed, the other two perpendicular velocities are assumed to be zero. The turbulent kinetic energy and its dissipation rate are assumed to be uniform and being calculated in terms of fixing the turbulence intensity value as $5 \%$. Boundary conditions for momentum transfer at all solid surfaces including the narrow and wide faces are specified with no slip boundary conditions. At the meniscus, a frictionless wall boundary condition mimicking the free surface of liquid steel is set. Similar boundary conditions are established for turbulent kinetic energy and its dissipation rate. Near any solid sur- 
face, a standard wall function for velocity distribution has been applied. At outlets, pressure outlet boundary condition is adopted. The boundary condition for electric potential $\phi$ at all surfaces is Newman with zero flux. ${ }^{13)}$ The density and dynamic viscosity of the molten steel are taken as $7200 \mathrm{~kg} /$ $\mathrm{m}^{3}$ and $0.00648 \mathrm{~kg} / \mathrm{m} . \mathrm{s}$, respectively. The electrical conductivity value of the liquid steel is used as $7140001 / \mathrm{ohm}-\mathrm{m}$ and Magnetic Permeability is set to $1.257 \times 10^{-6} \mathrm{~h} / \mathrm{m}$. Mathematical simulations of the process consist of employing a constant flow rate of incoming steel from the SEN (treated as with insulating walls) at a particular casting speed. The unsteady state simulations are performed with the combined flow, turbulence, and MHD Maxwell equations. ${ }^{14}$ It is essential to note that in order to analyze the electromagnetic breaking phenomena in continuous casting mold, assumption of solidifying steel shell to govern the electric current loop in the mold may be relevant. However, in the present investigation, we have neglected the formation of solidified shell ${ }^{13)}$ by considering the fact that non-conducting mold walls provide a return path for the induced electric current generated in the liquid steel. ${ }^{15}$ )

\subsection{Numerical Methodology, Grid Independence Test, and Model Validation}

Slab caster mold geometry and grid have been generated using commercial software Gambit. The governing MHD equations are solved using CFD software ANSYS Fluent, ${ }^{17}$ which uses the finite volume based technique. In the present work, a complete Hexahedral mesh has been generated with a quality of 0.85 (bad quality value $=0$; ideal quality value =1). A typical grid is shown in Fig. 1(a). The grid is refined very fine around the walls to capture near-wall turbulence. A grid independence study based on variation of the time averaged meniscus velocity has been carried out to choose the optimum grid points. For this purpose, simulations are performed at a casting speed of $1.25 \mathrm{~m} /$ min, section seize $1300 \mathrm{~mm} \times 218 \mathrm{~mm}$, and SEN submergence depth (SD) of $160 \mathrm{~mm}$. Figure 1(b) shows the results of grid independence study for four different mesh sizes, namely, 0.6 million, 1.1 million, 1.5 million, and
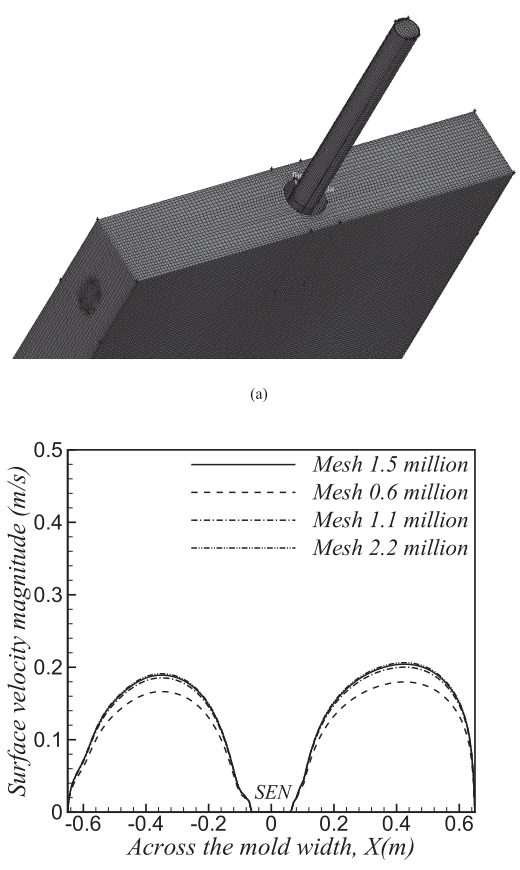

(b)

Fig. 1. (a) A typical grid used for computation. (b) Grid independence test results on time averaged meniscus velocity variation along the mold mid-plane at a casting speed of $1.25 \mathrm{~m} / \mathrm{min}$.
2.2 million, respectively. It can be seen from Fig. 1(b) that the grid independence situation has been achieved for 1.5 million cells, which is computationally economical for the present numerical investigation. The coupled momentum, turbulence, and MHD Maxwell equations have been solved using Semi Implicit Method for the Pressure Linked Equations (SIMPLE) algorithm. Furthermore, a second order upwinding scheme has been used in the convective terms. Appropriate grid density scheme has been adopted to capture small scale structures and fitting turbulence $y^{+}$value in a permissible range. To prevent numerical oscillations, the necessary conditions for time stepping have been determined from the Courant-Friedrichs-Lewy (CFL) condition.

For the purpose of validating present mathematical model with plant experimental result, nail board experiments ${ }^{14}$ ) have been performed at the continuous caster of Tata Steel LD2 plant. Typically for a casting speed of $1.25 \mathrm{~m} / \mathrm{min}$, the plant experimental measurement data of meniscus velocity variation at the mold mid-plane has been compared with the time averaged data of present mathematical model. The result is shown in Fig. 2(a), and can be seen to be in good agreement with the experimental data.

\section{Existing Current Settings and Magnetic Field Mea- surement}

For the case of EMBr, strength of the induced magnetic field depends on the percentage of current passing through the EMBr coils, both at the upper and lower coils (the present case). In general, the current settings vary with increasing casting speeds. The maximum current $(100 \%)$ is given beyond $1.5 \mathrm{~m} / \mathrm{min}$ casting speed. This is done by applying free charge resister to its maximum value. Detail circuiting arrangement can be found from ABB's manual. ${ }^{18)}$

In order to evaluate corresponding magnetic field intensity for different current settings, a Gauss meter measurement at plant has been carried out to obtain the profile
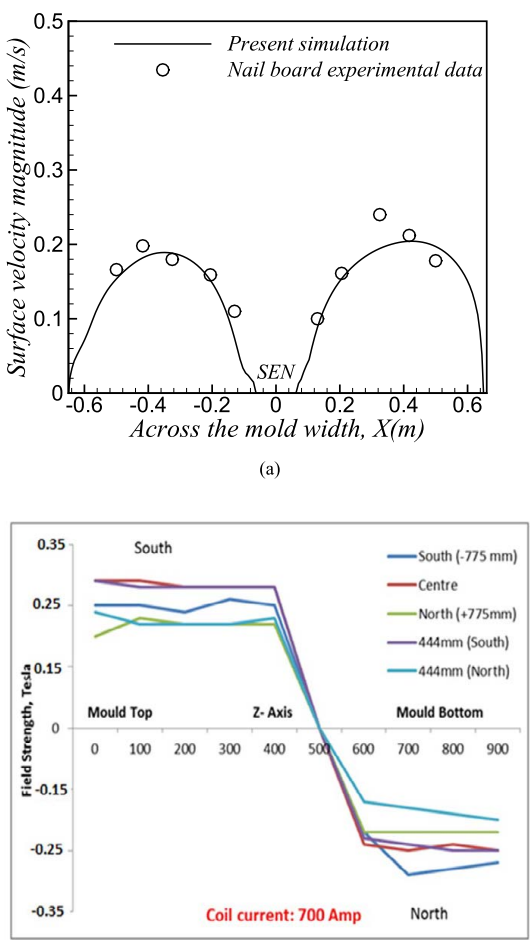

Fig. 2. (a) Comparison result of the time averaged meniscus velocity variation along the mold mid-plane at a casting speed of $1.25 \mathrm{~m} / \mathrm{min}$. (b) Typical magnetic field measurement data for $100 \%$ current settings, both at upper and lower coils. (Online version in color.) 
distribution of the magnetic field throughout the mold. For this 450 data point locations have been considered. Figure 2(b) displays the plant measurement data on the magnetic field strength along the mold. The data are shown at a typical situation of $100 \%$ current intensity case. It can be seen from Fig. 2(b) that there is a change in the sign of the magnetic field once it crosses the location downward from the meniscus at $500 \mathrm{~mm}$. This behaviour is quite obvious since there are two ruler type EMBr brake have been mounted on the mold. The lower brake produces a magnetic field which interacts with the magnetic field of the upper brake at the zone of neutrality, where its strength comes down to zero value. Furthermore, as can be seen from Fig. 2(b) that the field is positive at the upper brake, whereas it becomes negative for the lower brake.

\subsection{Distribution of Electromagnetic Field}

The initial magnetic field has been generated as per the measured gauss meter data for different current settings, both at Upper and Lower coils of the mold and the same field profile has been applied during mathematical modelling of the model continuous casting mold. Figures 3(a)-3(c) show the typical magnetic field distribution in the mold mid-plane for the current settings of: Upper coil $20 \%$, Lower coil 50\% (Fig. 3(a)); Upper coil 80\%, Lower coil 100\% (Fig. 3(b)); and Upper coil 100\%, Lower coil 100\% (Fig. 3(c)). The magnetic field is applied in terms of Tesla units. Corresponding to those magnetic fields (Fig. 3), the representative electric potential fields $(\phi)$ for casting speeds $1 \mathrm{~m} / \mathrm{min}, 1.3 \mathrm{~m} / \mathrm{min}$, and $1.5 \mathrm{~m} / \mathrm{min}$, are shown in Fig. 4(a).

It emerges from Fig. 3 that the zone of neutrality (the zone where magnetic field changes its sign) is below the

SEN ports. The electric potential contours, as shown in Fig. 4(a), reveals a cyclic rotation throughout the whole domain. This typical characteristic of electric potential brings an additional electromagnetic stress to that of fluid motion. It may be worth mentioning here that this electromagnetic stress is tensor quantity, and contains the classical Maxwell stress tensor that governs the electromagnetic interactions.

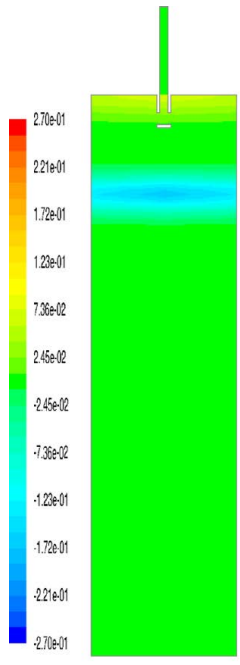

(a)

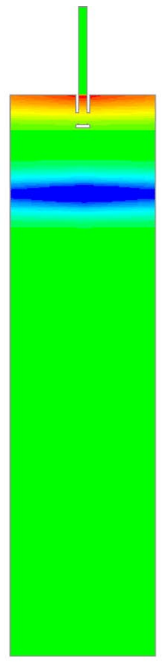

(b)

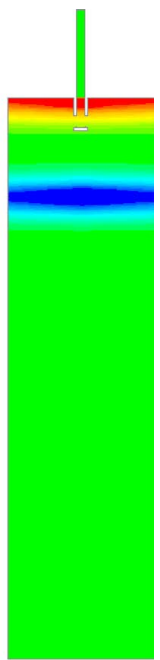

(c)

Fig. 3. Typical magnetic field distribution at the mold mid-plane at current settings (a) Upper coil 20\%, Lower coil 50\%, (b) Upper coil $80 \%$, Lower coil $100 \%$, (c) Upper coil $100 \%$, Lower coil $100 \%$. (Online version in color.)

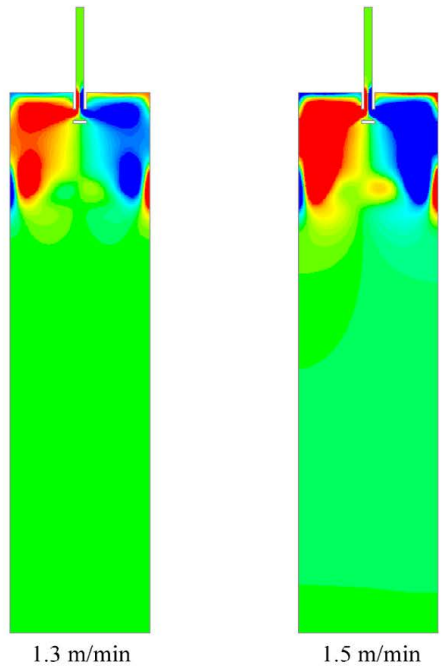

(a)

At meniscus

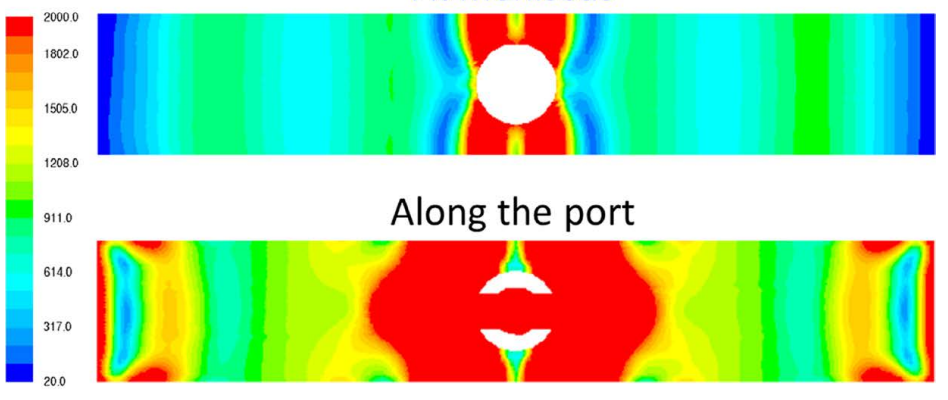

(b)

Fig. 4. (a) Electric potential field distribution at various casting speeds. (b) Lorentz force distribution at various locations. (Online version in color.) 
Accordingly, this causes a retarding motion to that of fluid inertial motion by satisfying constant global energy constraint. A lateral dampening effect is produced in the jet emanating from the port along the direction perpendicular to its propagation axis. This observation is quite similar to that of reported literature by Singh et al. ${ }^{13)}$ A typical double ruler type magnetic field distribution is evident along the mold plane. The electric potential plots also confirm typical double ruler brake type configuration. This is very typical to the Tata Steel caster EMBr and has been widely used at all such flow control mechanism in steel making processes. The electric potential, as shown in Fig. 4(a), has distinctive spread along the applied magnetic field plane. In addition, a cyclic loop of electric potential field is also evident along the port areas. In Fig. 4(b), we depict the distributions of Lorentz force contours at various cross sectional planes, namely, at the meniscus, at the port plane, of the mold. It is clear from Fig. 4(b) that the intensity of the Lorentz force is maximum at the port areas, analogously at the jet exit locations. The Lorentz force decays to its minimum value at the walls. The physical reason behind this typical behaviour may be offered as follows: at the port areas, the jet exit velocity is maximum and therefore generating higher magnitude of Lorentz force (since it is proportional to the $\left.U^{2} B_{0}\right)$. On the other hand, nearer to the mold walls, fluid velocity is small and therefore calling for lower Lorentz fore magnitude. Nevertheless, the magnitude of the Lorentz force at the port plane is always higher than that of meniscus.

\section{Results and Discussions}

The current work investigates the effect of superimposed static (DC) magnetic field (EMBr) on unsteady hydrodynamic characteristics of the slab caster mold at various casting speeds. Towards this, results are generated for situations both at with and without EMBr cases. For the simulation, the ranges of casting speeds from $1 \mathrm{~m} / \mathrm{min}$ to $1.8 \mathrm{~m} / \mathrm{min}$, in the steps of 0.1 have been considered. Apart from the regular section size of $1300 \mathrm{~mm}$, MHD simulations are carried out corresponding to two section sizes, namely, 1000 $\mathrm{mm}$ and $1500 \mathrm{~mm}$, respectively. Effects on changing ruler positions are performed for three variations of coil locations. For time marching simulations, iterations in each time loops are continued till global error in spatial domain reached to a limiting value, i.e. less than $10^{-4}$, whereas, in time domain, simulations have been performed till the system has reached a dynamically steady state situation (where hydrodynamic parameters varies asymptotically during time marching steps). The results are obtained after 400 seconds of simulation time. In the present work, the nozzle port angle has been fixed to a value of $15^{0}$ downward.

\subsection{Transient Fluid Flow and Turbulence Field}

Figures 5(a)-5(c) display the instantaneous contours
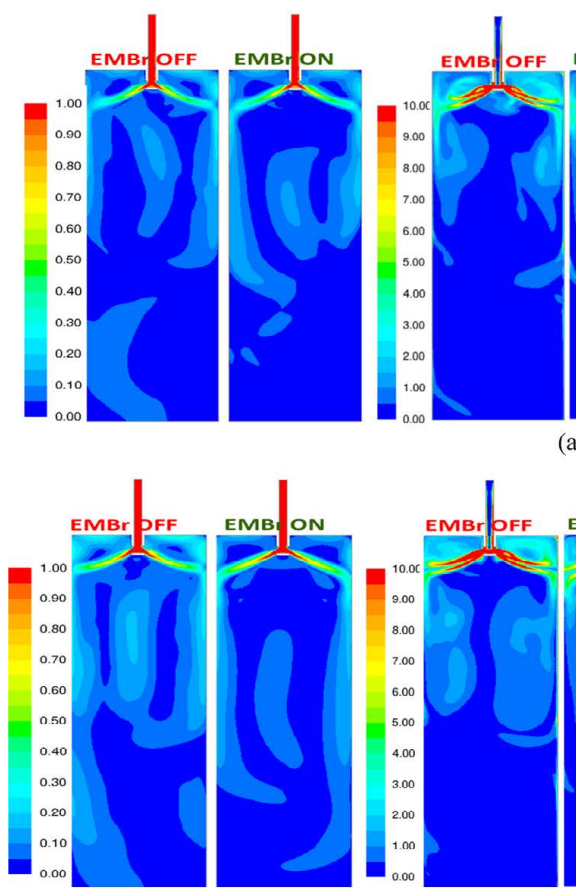
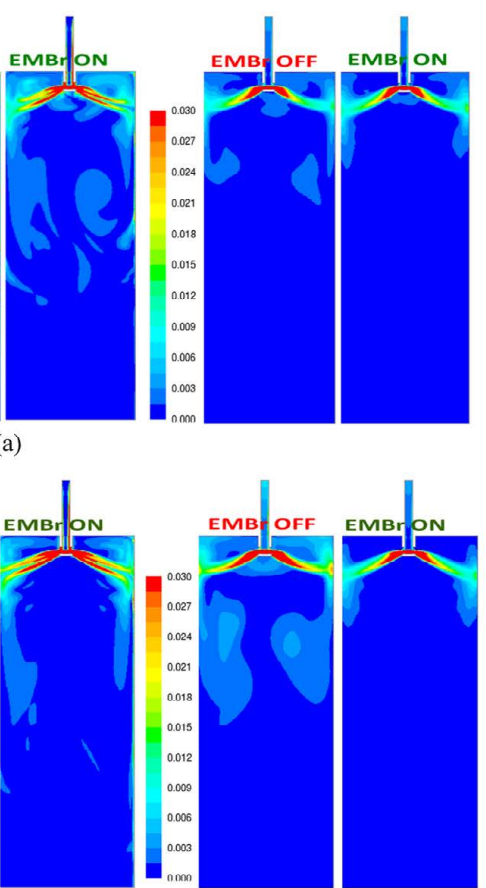

(b)
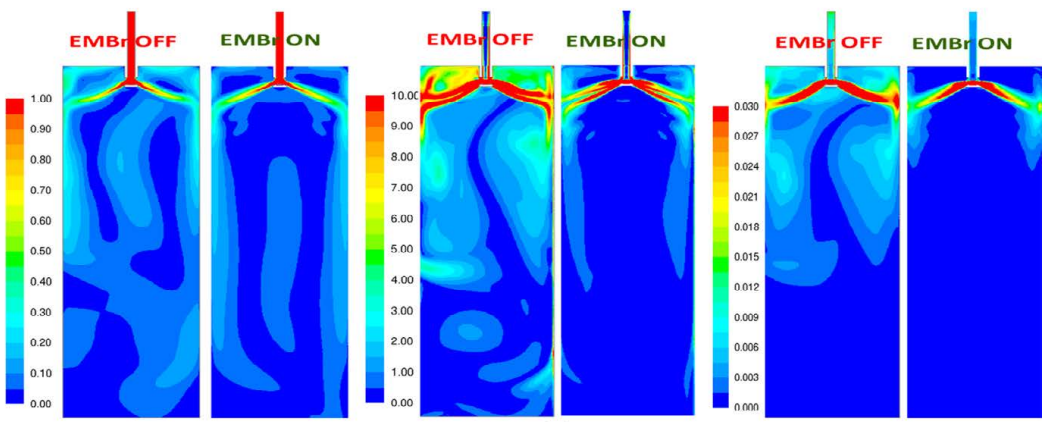

(c)

Contours of velocity

Contours of vorticity

Contours of turbulence $\mathrm{KE}$

Fig. 5. Instantaneous contours at the mold mid-plane for casting speeds (a) $1 \mathrm{~m} / \mathrm{min}$ (b) $1.3 \mathrm{~m} / \mathrm{min}$, (c) $1.5 \mathrm{~m} / \mathrm{min}$. (Online version in color.) 
of velocity, vorticity, and turbulence kinetic energy (KE) at the mold mid plane during EMBr OFF and EMBr ON situations at the three representative casting speeds of $1 \mathrm{~m} /$ min (Fig. 5(a)), $1.3 \mathrm{~m} / \mathrm{min}$ (Fig. 5(b)), and $1.5 \mathrm{~m} / \mathrm{min}$ (Fig. $5(c))$, respectively. The results are shown for a section size of $1300 \mathrm{~mm}$. As can be noticed, a typical "double-roll" type flow pattern in the mold corresponding to all the simulated cases is evident. ${ }^{19,20)}$ The liquid steel emanating from the SEN shows a strong flow directed across the top surface, toward the SEN from the narrow face, and deep into the strand the lower rolls penetrates. A typical observation can be drawn from the velocity contours is that the applied magnetic field produces a retarding effect in reducing heavy meniscus flow instabilities during EMBr OFF conditions. This observation is quite supportive with respect to that of vorticity contours. Vorticity field, signifying flow circulations, and corresponding shear layer instabilities, is found to be minimized with respect to that of EMBr OFF conditions. Furthermore, the turbulent $\mathrm{KE}$ is also found to be at calmer state in comparison to that of EMBr OFF condition. Pertinent to mention here is that in all such situations, the global effect of magnetic field in reducing flow instability is showing the marginal effect for the casting speed of $1 \mathrm{~m} / \mathrm{min}$ (Fig. 5(a)). A characteristic behaviour can be obtained from Fig. 5 that EMBr causes the jet to focus more while hitting the narrow face wall, reducing lateral dispersion, and directs the flow straight both at upward and downward directions respectively. In addition, thinning of the velocity boundary layer is also observed in the flow physics. Dramatic reduction in velocity fluctuations is also evident in the $\mathrm{EMBr}$ cases. Magnetic field suppresses turbulence and encourages the formation of large scale vertical structures in the mold whose axis align with the magnetic field. These large scale vertical structures move into the upper- and lowerrecirculation regions like laminar unsteady flows. This is the tendency towards quasi 2-D turbulence in the presence of a strong magnetic field and insulated walls normal to the magnetic field. ${ }^{13)}$ This behaviour is most dominant in the upper and lower recirculation regions. It can also be mentioned that in this condition, the viscous and magnetic dissipation of turbulence reduces and flow becomes dominated by large scale variations. ${ }^{14)}$ It is clear that, for the case of no EMBr, after the fluid flowing out of the SEN, the melt steel injects onto the narrow wall of the mold and then forms two big recirculation regions vertically in the mold, and the distribution of turbulent kinetic energy is spread. For the cases of $\mathrm{EMBr} \mathrm{ON}$, the vortices are controlled by the effect of the Lorentz force. As a result, the velocities around the SEN area dampen. Both of the vortex centres moves toward the SEN outlet; the vortex below the SEN shows a smaller drift, and the velocity changes its direction before impinging onto the narrow wall. Finally, the homogeneous flow appears in the lower part of the mold, indicating an obvious suppression of the turbulence intensity.

A critical remark can be made at this juncture by comparing the vorticity dynamics in the mold with the analogy for the flow past a circular cylinder. It is a well-known fact that for the flow past a circular cylinder, a spatio-temporal intermittency in the flow domain creates instability which initiates the formation of coherent vortex blob that separates from its own source due to the mechanism of accumulation and constriction process. As a result, a periodic coherent vortex structure is found to shed in the bluff body wake, which is legendarily known as von Ka'rma'n vortex street. This phenomenon can be easily visualized from the vorticity contours, where a separated coherent vortex blob is evident nearer to the mold outlet (Fig. 5(c)). Occurrence of this phenomena nearer the SEN signifies an unbalanced flow among the sides of the mold. Furthermore, these vortices at the mold surface can cause entrapment a funnel of molten slag into the liquid steel if it is going together with the downward pull of the primary metal flow. For sufficiently large height of this slag funnel may get closer to the jetting region. ${ }^{13,21)}$ As a result, it become fragmented into droplets and gets entrained into the jet. This causes entrapment of the slag into the final product. It is observed that with $\mathrm{EMBr}$ ON cases, there is an overriding suppression of this vortex shedding effect and therefore ensuring improvement in the final cast quality. ${ }^{14)}$

\subsection{Top Surface Stabilization}

We proceed further in analysing what is happening to the fluctuations in meniscus velocity vector during $\mathrm{EMBr}$ $\mathrm{OFF}$ and $\mathrm{ON}$ conditions respectively. In general, meniscus level stabilization can qualitatively be judged by the velocity vectors at the meniscus. Meniscus stability determines the order of the defect in the final cast products. A departure from meniscus stability gives rise to the formation of standing waves, which forms from the flow of steel beneath the free surface. A high local slope of the flow triggers instability of the meniscus and as a result a heavy meniscus level fluctuation (commonly referred as MLF) is observed. Figures 6(a)-6(c) show the velocity vector at the meniscus for $\mathrm{EMBr} \mathrm{OFF}$ and $\mathrm{ON}$ conditions at casting speeds of $1 \mathrm{~m} /$ min (Fig. 6(a)), $1.3 \mathrm{~m} / \mathrm{min}$ (Fig. 6(b)), and $1.5 \mathrm{~m} / \mathrm{min}$ (Fig. $6(\mathrm{c}))$, respectively. The results are presented at a representative section size of $1300 \mathrm{~mm}$. A clear departure from the meniscus stabilization is noticed in Fig. 6 at the EMBr OFF conditions. The meniscus becomes locally turbulent

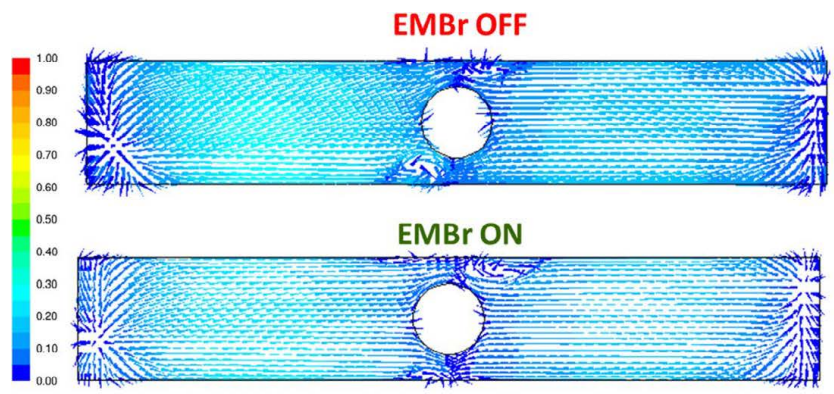

(a)

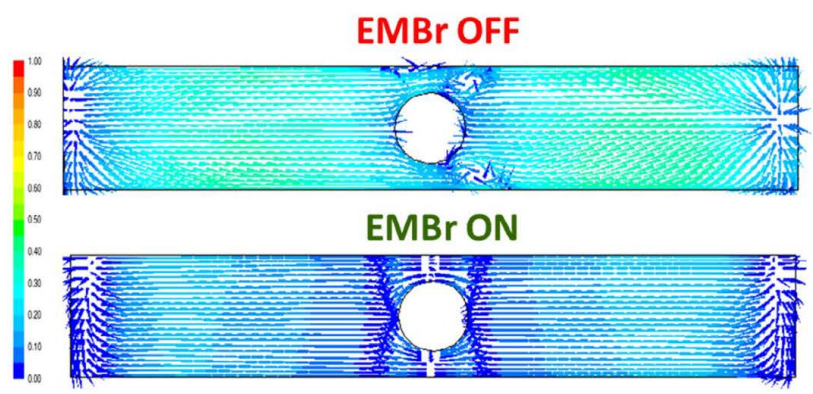

(b)

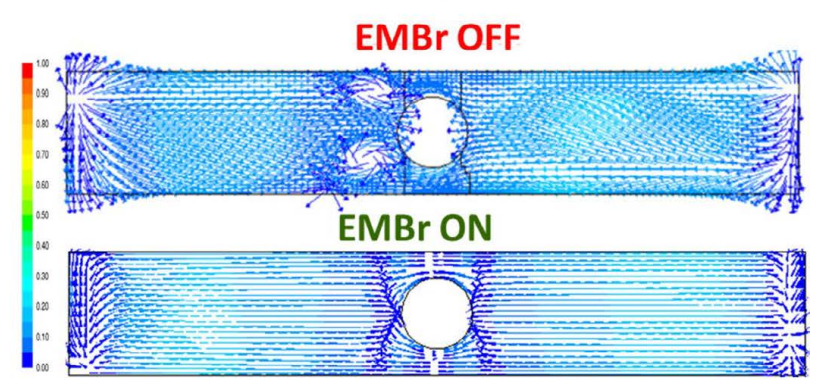

(c)

Fig. 6. Instantaneous velocity vectors at the meniscus for casting speeds (a) $1 \mathrm{~m} / \mathrm{min}$ (b) $1.3 \mathrm{~m} / \mathrm{min}$, (c) $1.5 \mathrm{~m} / \mathrm{min}$. (Online version in color.) 
and formation of strong re-circulation zone is noticed. Such high turbulence at the meniscus imparts heavy mold level fluctuations and may cause the solidifying dendritic shell expose to the slag layer. This in effect forms sliver just underneath the meniscus and the associated sliver defect in the final cast product. ${ }^{14)}$ In contrast, it can be noticed from Fig. 6 that EMBr ON condition imparts meniscus stabilization and significantly suppressed meniscus level disturbance. This, in turn, reduces the formation of localized circulatory loops nearer to the SEN area. The meniscus is calmer, surface vectors point along the SEN direction and is parallel to each other. However, a small recirculation zone is observed nearer to the SEN area and this is because

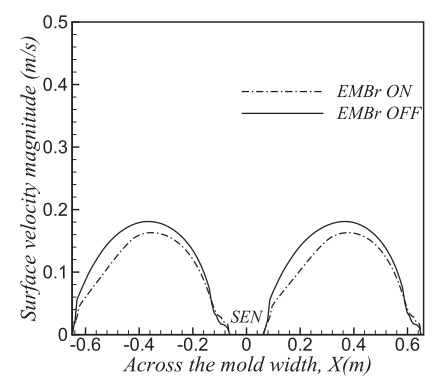

(a)

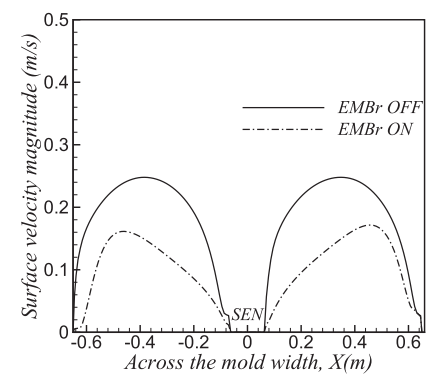

(c)

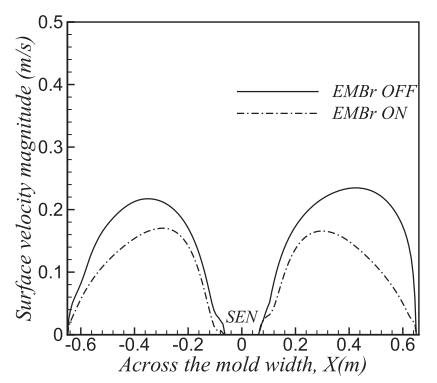

(b)

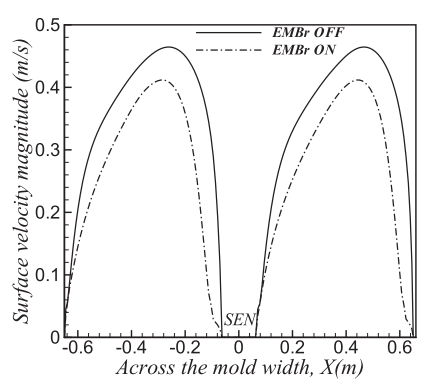

(d) of swirling phenomena arising out of high jet impacting velocity. Application of magnetic field suppresses both the small and large scale fluctuations, resulting in stable surface behaviour. ${ }^{13)}$ Towards this, it is appropriate to mention here that the Lorentz force (per unit volume) of magnitude $\vec{j} \times \vec{B}$ in the flow domain is generated by the magnetic field, which acts as an impeding influence to the resultant axial transport. The magnitude of this magnetic field induced retardation is proportional to the square of the magnetic field $\left(\sim B_{Y}^{2}\right)$, and flow strength $(U)$. This, in turn, finally causes turbulence suppression, as the turbulence intensity magnitude is directly proportional to the local fluid velocity magnitude.

\subsection{Effect on Time-averaged Velocity Variation at the Meniscus Mid Plane}

In this section the effect of magnetic field in reduction of meniscus mid plane velocity variation is quantitatively analyzed. For this purpose, results are shown with the timeaveraged value of meniscus- mid plane velocity values. For a section size of $1300 \mathrm{~mm}$, Figs. 7(a)-7(d) display the time-averaged velocity variation at the meniscus mid-plane for EMBr OFF and ON conditions at casting speeds of $1 \mathrm{~m} /$

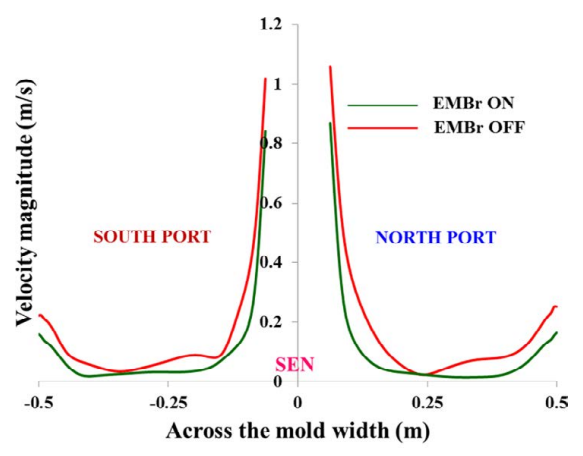

(a)

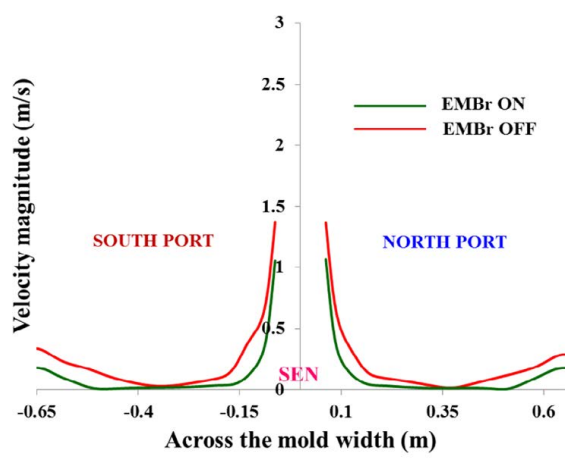

(b)

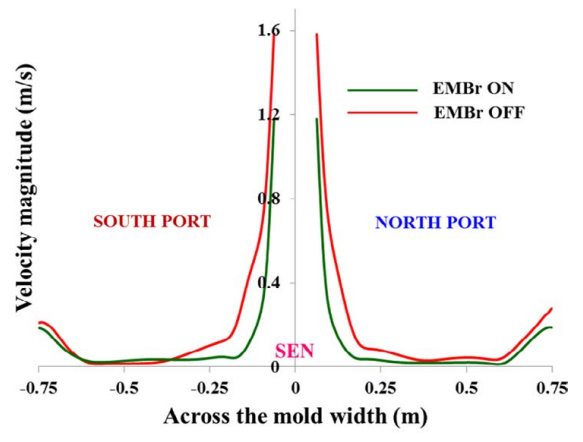

(c)

Fig. 9. Time-averaged velocity variation along the port plane during EMBr OFF and ON conditions for section sizes (a) 1000 $\mathrm{mm}$, (b) $1300 \mathrm{~mm}$, (c) $1500 \mathrm{~mm}$. (Online version in color.) 
min (Fig. 7(a)), $1.3 \mathrm{~m} / \mathrm{min}$ (Fig. 7(b)), $1.5 \mathrm{~m} / \mathrm{min}$ (Fig. 7(c)), and $1.8 \mathrm{~m} / \mathrm{min}$ (Fig. 7(d)), respectively. At a representative casting speed of $1.4 \mathrm{~m} / \mathrm{min}$, Figs. 8(a) $-8(\mathrm{~b})$, illustrates the variation of the time-averaged velocity at the meniscus midplane for $\mathrm{EMBr} \mathrm{OFF}$ and $\mathrm{ON}$ conditions at section sizes of $1000 \mathrm{~mm}$ (Fig. 8(a)) and $1500 \mathrm{~mm}$ (Fig. 8(b)). It can be seen from Figs. 7-8 that the effect of EMBr dampens meniscus velocity than that of without EMBr case. Typical for a situation of $1 \mathrm{~m} / \mathrm{min}$ casting speed (Fig. 7(a)), the maximum percentage reduction in meniscus velocity is no more than $2 \%$. Therefore, this is also supports our earlier observation that at this low casting speed the imposition of $\mathrm{EMBr}$ is not so effective, since the current percentage is low and therefore inducing lower magnetic field strength. Furthermore, for casting speed range $1.3 \mathrm{~m} / \mathrm{min}-1.5 \mathrm{~m} / \mathrm{min}$ (Figs. 7-8), significant reduction in meniscus velocity is noticed. This is a welcome result, since a proper choice of magnetic field and thereby current settings for a typical casting speed can be made from this observation. In this context, it may be mentioned that the decrease of meniscus velocity is a direct consequence of the suppression of the turbulence in the flow. This is attributed to an average 30\% decrement in the maximum turbulence intensity with $\mathrm{EMBr}$ on conditions, which, as obtained from the present computational results. It has been discussed in the preceding section that steel quality is a direct function of the surface flow speed. Due to the mechanism of shear-layer instability, higher magnitude of the meniscus velocity prone to slag entrainment, whereas meniscus freezing may occur if the surface velocity is lower in magnitude. ${ }^{19)}$ Therefore, selection of the safe operating window between the higher and lower threshold is necessary to avoid those effects. It has been reported in the literature that the ideal range of mold top surface velocity should vary in between $0.26 \mathrm{~m} / \mathrm{s}$ to $0.43 \mathrm{~m} / \mathrm{s}$, perhaps the appropriate value varies from the plant to plant and will depend on the superheat and other online plant operating conditions. ${ }^{22)} \mathrm{A}$ typical observation can be noticed from Figs. 7-8 that the distribution of time-averaged velocity is somewhat stretched and generally do not follow an approximate parabolic profile. This behaviour is due to complex interplay between magnetic field induced Lorentz forced, liquid steel velocity, and the turbulent large scale structures. ${ }^{13)}$ Double ruler magnetic brake configurations are also responsible for this behaviour, since the zone of neutrality encourages turbulent structures to dissipate faster.

\subsection{Effect on Time-averaged Velocity Variation along the Port Plane}

To investigate further the effect of EMBr on the velocity reduction along the jet area, a representative location is primarily selected along the port plane (at $0.225 \mathrm{~m}$ below the meniscus). At a representative casting speed for 1.4 $\mathrm{m} / \mathrm{min}$, Figs. 9(a)-9(c) show the time-averaged velocity variation along the port plane during EMBr OFF and ON
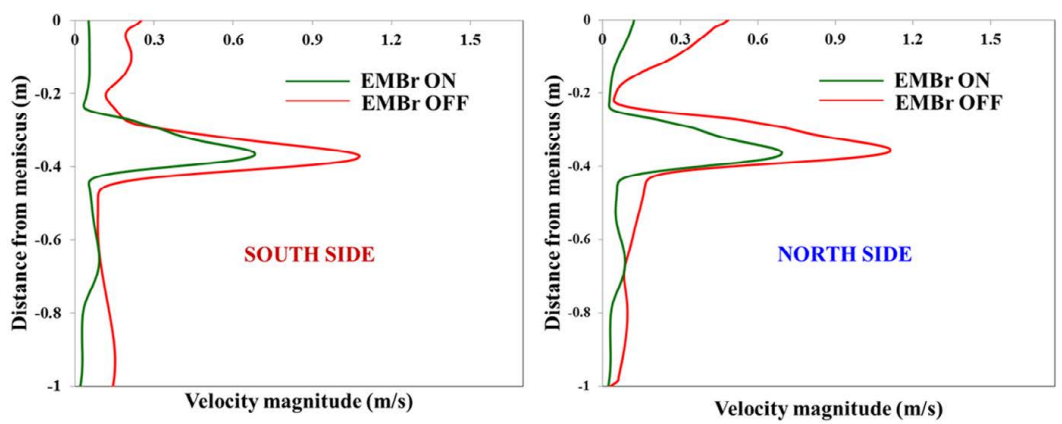

(a)
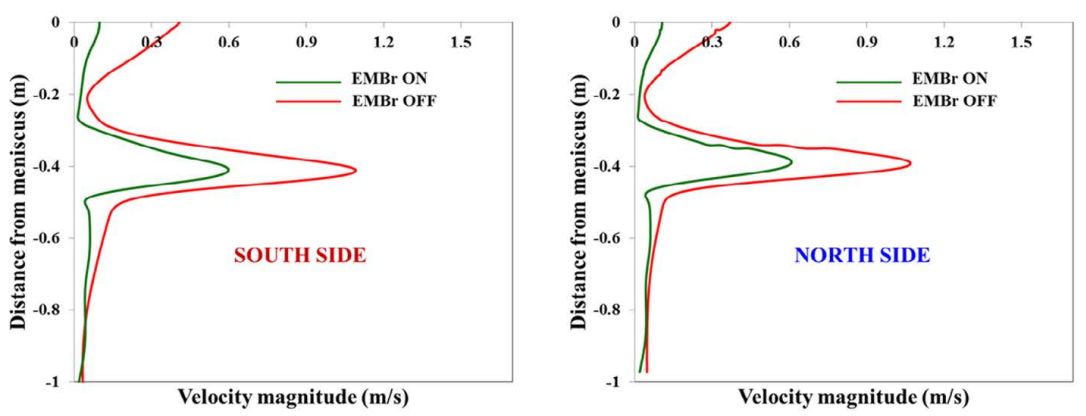

(b)
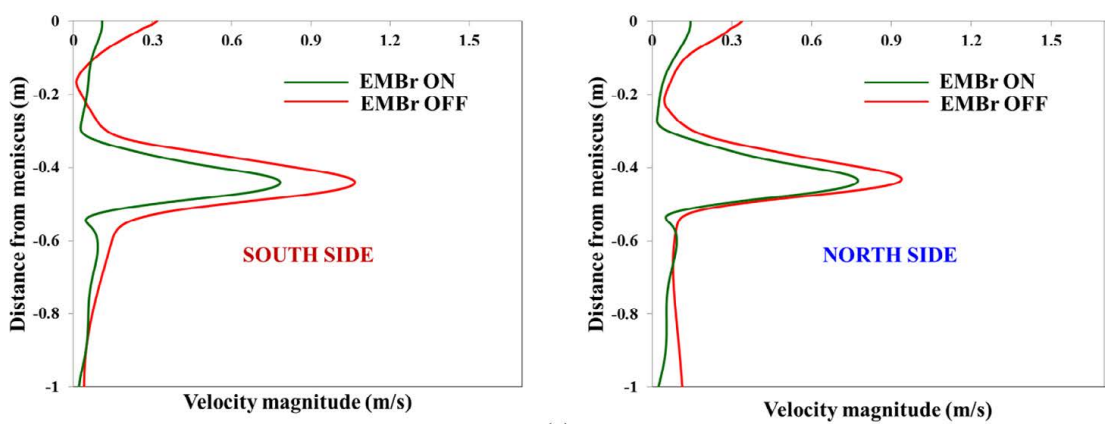

(c)

Fig. 10. Time-averaged velocity variation along the casting direction at section sizes (a) $1000 \mathrm{~mm}$, (b) $1300 \mathrm{~mm}$, (c) $1500 \mathrm{~mm}$. (Online version in color.) 
conditions for section sizes $1000 \mathrm{~mm}$ (Fig. 9(a)), $1300 \mathrm{~mm}$ (Fig. 9(b)), and $1500 \mathrm{~mm}$ (Fig. 9(c)). It is noticed from Fig. 9 that the velocity differences between EMBr OFF and ON conditions are dampened throughout the whole section sizes. It is observed that the there is a sudden jump of the time averaged velocity in the port areas. This is because of the fact that the jet is exiting from the ports in that location and therefore sudden jump in the velocity magnitude. Overall trend of the velocity profile shows that the variation is symmetric and the maximum velocity magnitude is observed along the port area, which further decays down as one move towards the narrow face of the mold. This has a consequence in suppression of the meniscus velocity, as the mean velocity of the spouting stream from the SEN is also suppressed. It is worth mentioning here that the jet penetration depth enhances with increasing downward velocity beneath the jet area, as a result, casing inclusion to be arrested into the solidified shell. ${ }^{14}$ )

\subsection{Effect on Time-averaged Velocity along the Cast- ing Direction}

To investigate further the effect of EMBr on the velocity suppression along the casting direction (downward direction from the meniscus), two representative locations are chosen at the mold mid plane at $x= \pm 0.325 \mathrm{~m}$. The downward directional length below the meniscus is taken till $z=-1$ $\mathrm{m}$, thus covering the jet area. At a fixed casting speed of 1.4 $\mathrm{m} / \mathrm{min}$, Figs. 10(a)-10(c) show the time-averaged velocity variation along the downward direction from the meniscus till $z=-1 \mathrm{~m}$ and at locations $x=-0.325 \mathrm{~m}$ (South side of the mold) and $x=0.325 \mathrm{~m}$ (North side of the mold), for section sizes $1000 \mathrm{~mm}$ (Fig. 10(a)), $1300 \mathrm{~mm}$ (Fig. 10(b)), and $1500 \mathrm{~mm}$ (Fig. 10(c)), respectively. It can be found from Fig. 10 that the overall variation of the time averaged velocity is symmetric and it grossly dampens from the port region. This is probably because of the reason that there is an early laminarization of the flow in the SEN by the upper ruler during inflowing to the mold followed by a turbulent zone reaching the bottom of the nozzle situating at the weaker magnetic field zone. ${ }^{14)}$ Pertinent to mention here that towards the meniscus direction, velocity variation is slightly higher. This is due to the shearing action of mold level velocity towards the SEN direction.

\subsection{Effect on Changing Ruler Positions}

To further reveal how the applied magnetic field suppresses the mold turbulence and global flow dynamics by changing the positions of EMBr rulers, simulations are carried out for three consecutive sets with respect to the base case of existing coil positions. The assumed cases are (i) Upper ruler moved below to $0.1 \mathrm{~m}$ from the existing location; Lower ruler moved up to $0.1 \mathrm{~m}$ from the existing location, ii) Upper ruler moved below to $0.1 \mathrm{~m}$ from the existing location; Lower at the existing location, and iii) Upper ruler at existing location; Lower ruler moved up to $0.1 \mathrm{~m}$ from the existing location. At a fixed section size of $1300 \mathrm{~mm}$, simulations are performed for a representative casting speed of $1.4 \mathrm{~m} / \mathrm{min}$. The results are generated for all the cases and compared with the EMBr OFF solution. At a representative casting speed of $1.4 \mathrm{~m} / \mathrm{min}$, Figs. 11(a) 11(b) show the comparison results on the instantaneous contours of velocity (Fig. 11(a)), vorticity (Fig. 11(b)) at the mold mid plane for EMBr OFF, existing, and the chosen three cases, respectively. It can be inferred from Fig. 11 that a change in ruler position changes flow dynamics in the upper part of the mold significantly. Such phenomena are predominant for Case (i) and Case (ii). The plausible reason behind this behaviour is due to changing magnetic flux intensity distribution in the upper part of the mold. For these cases the magnetic field induced Lorentz force
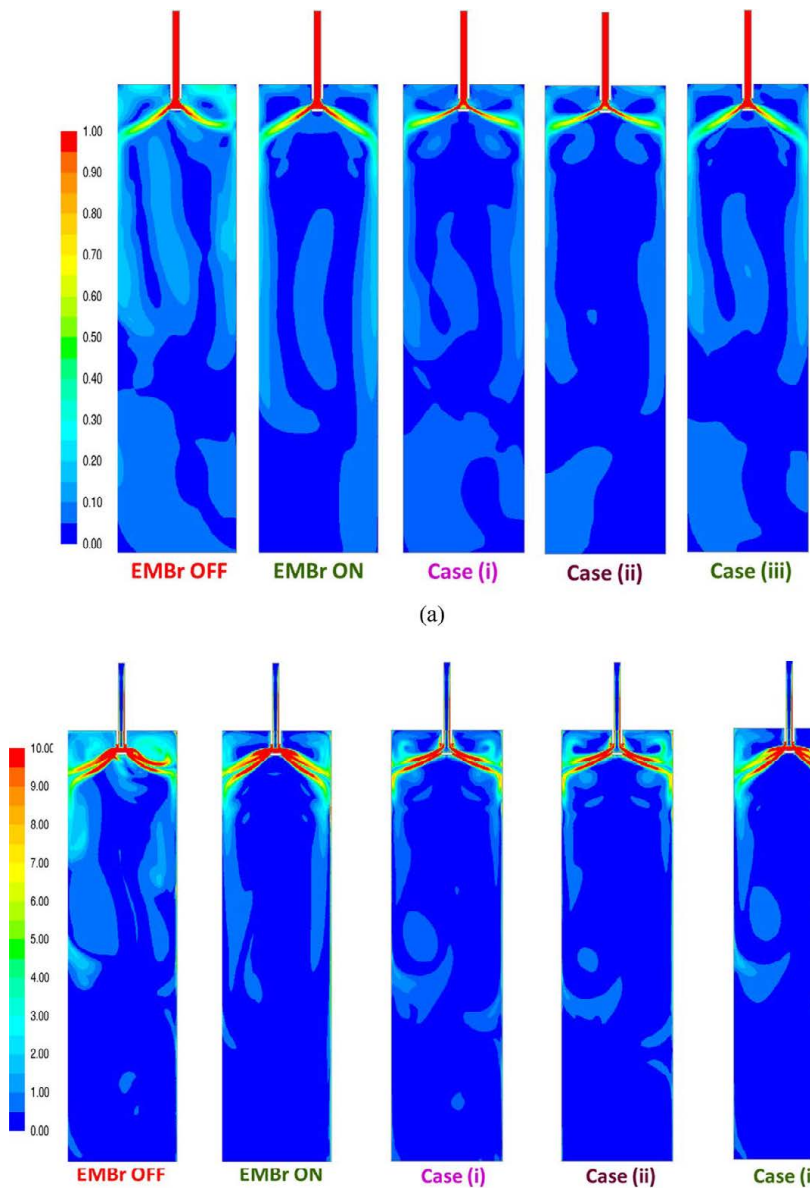

(a)
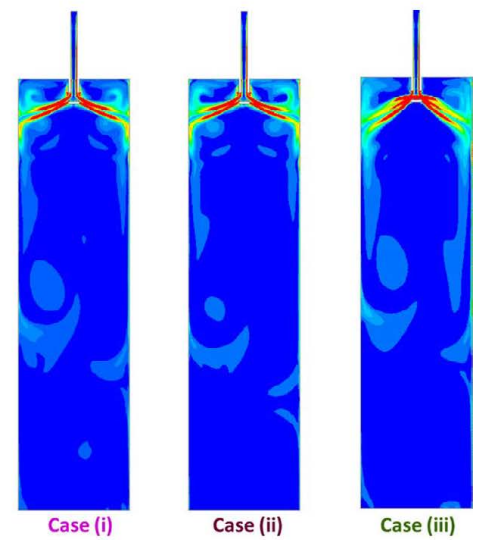

(b)

Fig. 11. Comparison results on the instantaneous contours of (a) velocity and (b) vorticity field at the mold mid plane for EMBr OFF, existing, and three cases. (Online version in color.)

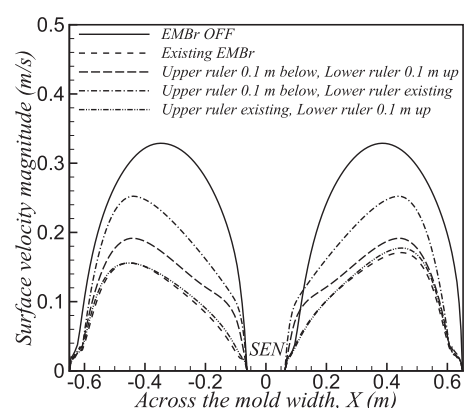

Fig. 12. Time averaged meniscus velocity variation at the mold mid plane for EMBr OFF and three different Cases at a casting speed of $1.4 \mathrm{~m} / \mathrm{min}$.

directly interacts with the jets emanating from the ports and causes the early displacement towards the meniscus region. On the other hand, the relative intensity of the magnetic field nearer to the meniscus is feeble and therefore does not have sufficient strength in displacing the liquid steel in the opposite direction. As a result, global transport due to fluidic inertia dominates over the magnetohydrodynamic transport of the flow. In the upper part of the mold, a large scale recirculation alternating between the north and south sides of the mold is noticed. There is a flip-flop motion of the fluid along the narrow mold face is also noticed during subsequent instances of the transience. ${ }^{14)}$

A further quantification on the global reduction in meniscus velocity in changing coil positions has been done by plotting the time-averaged meniscus velocity in the mold mid-plane for various Cases. Figure 12 compares the time 
averaged meniscus velocity in the mold mid plane for different Cases and EMBr OFF condition, at a casting speed of $1.4 \mathrm{~m} / \mathrm{min}$. It is interesting to note from Fig. 12 that in comparison to that of all other Cases, the existing coil position displays the minimum reduction in the meniscus velocity. Notably, similar behaviour with that of existing coil position is evident only for the Case (iii), where the upper coil is at existing position and lower coil is moved up to $0.1 \mathrm{~m}$. It is therefore recommended not to tailor the exiting ruler position in order to achieve the desired meniscus velocity. It is pertinent to mention here that the above study on changing ruler positions is a representative case and similar results are also noticed for other casting speeds and section sizes.

\section{Summary and Conclusions}

In the present investigation, a three dimensional magnetohydrodynamic flow model for the slab caster has been developed by coupling fluid flow equations and Maxwell equations. The major innovation of this work is the development of a validated MHD model for slab caster which is the first time at Tata Steel India. The model evaluates global flow dynamics under the effect of externally applied magnetic field at different current settings for various casting speeds. The simulation result exhibits the following conclusions:

- Superimposed magnetic field suppresses turbulence, resulting in a decrease of meniscus velocity. The resulting jets exiting from the ports are straight, thin, and laminarized. The region of the strongest magnetic field tends to deflect the jet away. This leads to more upward directed flow.

- Symmetricity in velocity distribution along the SEN and the jet plane is noticed. Existing magnetic field less than $1.3 \mathrm{~m} / \mathrm{min}$ casting speed does not have any significant effect on reduction in overall velocity in the mold. Analogous effect is also noticed at $1.8 \mathrm{~m} / \mathrm{min}$ casting speed.

- Effect of changing section sizes to $1000 \mathrm{~mm}$ and $1500 \mathrm{~mm}$ show similar trend to that of $1300 \mathrm{~mm}$. A slightly higher reduction in turbulence and meniscus velocity is seen at $1500 \mathrm{~mm}$ section size, where the thinning of jet is found to be higher.

- Moving the Upper ruler downward (Case (i) and (ii)) deflects the jet upward, leading to a shallower downward jet angle and higher surface velocity. Moving Lower ruler upward (Case (iii)) have minimal effect in meniscus velocity reduction.

- Positioning the strongest magnetic fields across the nozzle port regions leads to detrimental unstable flow, so this practice should be avoided. Similar effect is seen at higher casting speed $(1.5 \mathrm{~m} / \mathrm{min})$.

\section{Acknowledgments}

The author would like to acknowledge Dr. Rajneesh Chaudhary, ATC, Pennsylvania, USA, for useful technical discussions. The author would also like to thank Prof. Eiki KASAI, Editor in Chief, ISIJ International, for kind considerations in reconsidering the manuscript for resubmission.

\section{REFERENCES}

1) B. G. Thomas and L. Zhang: ISIJ Int., 41 (2001), 1181.

2) L. C. Hibbeler, R. Liu and B. G. Thomas: Proc. 7th European Conf. on Continuous Casting, The Institute of Materials, Minerals and Mining, Düsseldorf, (2011).

3) K. Timmel, X. Miao, S. Eckert, D. Lucas and G. Gerbeth: Magnetohydrodynamics, 46 (2010), 337.

4) K. Timmel, S. Eckert and G. Gerbeth: Metall. Mater. Trans. B, 42 (2011), 68 .

5) K. Okazawa, T. Takehito, J. Fukuda, T. Kawase and M. Toki: ISIJ Int., 41 (2001), 851.

6) P. H. Dauby and S. Kunstreich: Ironmaking Steelmaking, 32 (2005), 80.

7) Y. Hwang, P. Cha, H. Nam, K. Moon and J. Yoon: ISIJ Int., 37 (1997), 659.

8) Q. Yuan, S. Sivaramakrishnan, S. P. Vanka and B. G. Thomas: Metall. Mater. Trans. B, 35 (2004), 967.

9) B. Zhao, B. G. Thomas, S. P. Vanka and R. J. O'Malley: Metall. Mater. Trans. B, 36 (2005), 801.

10) Z.-D. Qian and Y.-L. Wu: ISIJ Int., 44 (2004), 100.

11) R. Kageyama and J. W. Evans: ISIJ Int., 42 (2002), 170.

12) Y. Miki and S. Takeuchi: ISIJ Int., 43 (2003), 1548.

13) R. Chaudhary, B. G. Thomas and S. P. Vanka: Metall. Mater. Trans. $B, 43$ (2012), 532.

14) R. Singh, B. G. Thomas and S. P. Vanka: Metall. Mater. Trans. B, 45 (2014), 1098.

15) R. Moreau: Magnetohydrodynamics, Kluwer Academic Pub. Co., Norwell, MA, (1990), 50.

16) S. B. Pope: Turbulent Flows, Cambridge University Press, Cambridge, UK, (2000), 45.

17) Fluent Inc.: ANSYS FLUENT 15.0 User's Guide, Fluent Inc., Lebanon, NH, (2015).

18) ASEA Brown Boveri (ABB): EMBR for slab casters, Boosting Productivity and Quality in SlabCasting with ABB's Electromagnetic Systems, ABB Inc., Zurich, (2007).

19) K. Timmel, S. Eckert, G. Gerbeth, F. Stefani and T. Wondrak: ISIJ Int., 50 (2010), 1134.

20) R. Liu, W. Ji, J. Li, H. Shen and B. Liu: Steel Res. Int., 79 (2008), 50.

21) R. Chaudhary, C. Ji, B. G. Thomas and S. P. Vanka: Metall. Mater. Trans. B, 42 (2011), 987.

22) L. C. Hibbeler and B. G. Thomas: AISTech Steelmaking Conf. Proc., Warrendale, PA, (2013). 